Document downloaded from:

http://hdl.handle.net/10251/78690

This paper must be cited as:

Castiñeira lbáñez, S.; Rubio Michavila, C.; Sánchez Pérez, JV. (2015). Environmental noise control during its transmission phase to protect buildings. Design model for acoustic barriers based on arrays of isolated scatterers. Building and Environment. 93:179-185. doi:10.1016/j.buildenv.2015.07.002.

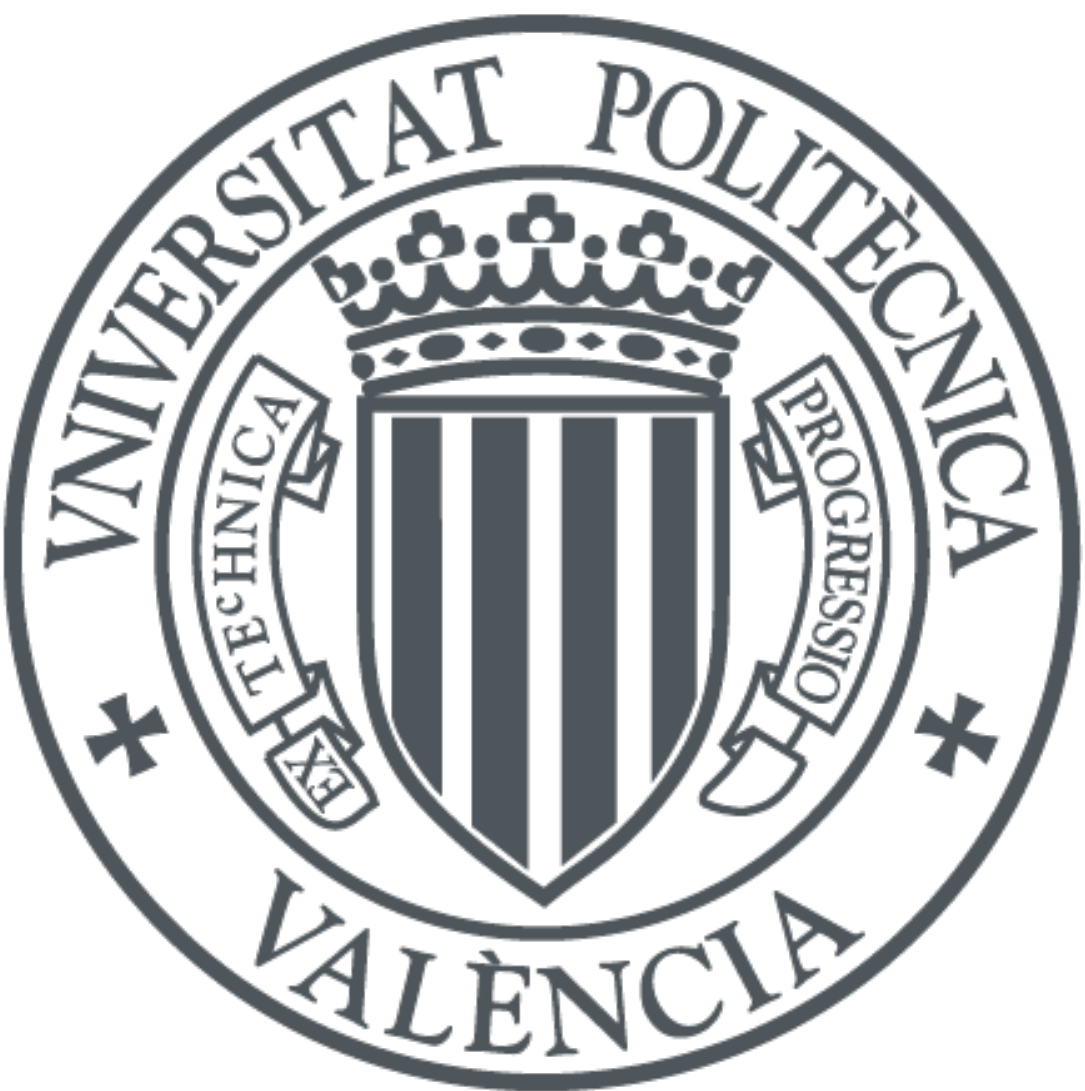

The final publication is available at

http://dx.doi.org/10.1016/j.buildenv.2015.07.002

Copyright Elsevier

Additional Information 


\section{Accepted Manuscript}

Environmental noise control during its transmission phase to protect buildings. Design model for acoustic barriers based on arrays of isolated scatterers

Sergio Castiñeira-Ibañez, Constanza Rubio, Juan Vicente Sánchez-Pérez

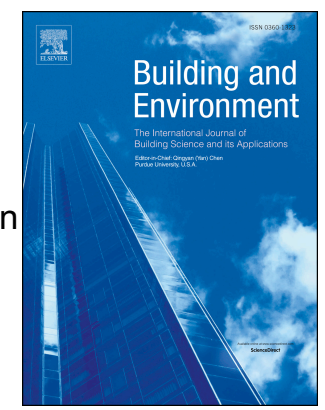

PII:

S0360-1323(15)30051-2

DOI:

10.1016/j.buildenv.2015.07.002

Reference: BAE 4180

To appear in: Building and Environment

Received Date: 30 March 2015

Revised Date: 20 June 2015

Accepted Date: 4 July 2015

Please cite this article as: Castiñeira-lbañez S, Rubio C, Sánchez-Pérez JV, Environmental noise control during its transmission phase to protect buildings. Design model for acoustic barriers based on arrays of isolated scatterers, Building and Environment (2015), doi: 10.1016/j.buildenv.2015.07.002.

This is a PDF file of an unedited manuscript that has been accepted for publication. As a service to our customers we are providing this early version of the manuscript. The manuscript will undergo copyediting, typesetting, and review of the resulting proof before it is published in its final form. Please note that during the production process errors may be discovered which could affect the content, and all legal disclaimers that apply to the journal pertain. 


\title{
ENVIRONMENTAL NOISE CONTROL DURING ITS TRANSMISSION PHASE TO PROTECT BUILDINGS. DESIGN MODEL FOR ACOUSTIC BARRIERS BASED ON ARRAYS OF ISOLATED SCATTERERS.
}

Sergio Castiñeira-Ibañez, Constanza Rubio*, Juan Vicente Sánchez-Pérez

Centro de Tecnologías Físicas: Acústica, Materiales y Astrofísica. División Acústica.

Universitat Politècnica de València. Camino de Vera s/n. 46022 Valencia, Spain.

Phone: 34.963879521; Fax: 34.963879525; e-mail: crubiom@fis.upv.es

*Corresponding author.

\begin{abstract}
A new generation of acoustic barriers is being introduced into the noise control market. These barriers, based on arrays of isolated scatterers, present interesting properties to be used in cities to reduce the transmitted transport noise affecting buildings. Among them, both aesthetic and continuity factors of the urban landscape may be mentioned. This new kind of barrier is technologically advanced and acoustically competitive with respect to the current ones formed by continuous rigid materials. To design these barriers whilst taking into account their inherent acoustic complexity, we present an overlapping numerical model here that enables us to split the real three-dimensional problem into two two-dimensional ones, allowing both the reduction of the computational cost and the separate analysis of each one of the noise control mechanisms involved. We analyse different cases, checking the numerical simulations with accurate experimental results.
\end{abstract}

Keywords: Sonic Crystal, Acoustic Barrier, Numerical Model, Noise Control in transmission, 


\section{NTRODUCTION}

Noise, defined as an unwanted or unpleasant outdoor sound generated by human activity, is one of the main environmental problems all over the world [1]. Noise pollution is especially important in places where people carry out their activities. The case of the cities is particularly interesting, where a conflict of interests is presented: on one hand, high noise levels are created because of the existence of human activities and, on the other hand, low noise levels are necessary to enable people to rest. The composition of urban noise is complex due to the superposition of a great variety of sounds which are originated by different sources and is therefore characteristic of each particular area. The complete range of sounds that characterise a city is usually called urban soundscape and was introduced by Schafer [2]. In every case, the main contribution to the urban soundscape is supplied by noise transport (road traffic, aircraft and railway noise), and the rest of the noise components (ranging from the ringing of a bell to the conversation of a group of people on the street) are added to this main noise source. So, when we are talking about acoustic comfort in buildings it would be interesting to discuss how to reduce transport noise in cities.

Generally speaking, noise control can be carried out in each of the three phases into which noise propagation may be divided: (i) noise generation at the source; (ii) transmission of noise from source to receiver and (iii) noise reception. In the case of transport noise, the best and cheapest solution is to act on the noise source, reducing its levels by means of the implementation of certain actions such as reduction of vehicles speed or the use of noiseabsorbing pavements. On the other hand, as regards noise reception, we have to consider sound insulation of buildings. Although it seems quite a good strategy in the case of new buildings, noise insulation cost is much higher when we refer to the case of old dwellings. 
Moreover, these previously mentioned noise control actions can only be applied to control the inside of houses, thus preventing the enjoyment of outdoor areas such as gardens or terraces.

The last action framework in noise control is to act in the phase of noise transmission from source to receiver, i.e. from the noise created in the streets by transport all the way to the buildings, including their outdoor recreational areas. The main existing tool to reduce noise in this phase is the use of acoustic barriers (ABs), defined as a continuous rigid material with a minimum superficial density of $20 \mathrm{~kg} / \mathrm{m}^{2}$ [3]. The acoustic effect of $\mathrm{ABs}$ can be explained as follows: the transmitted noise travels from the source to the receiver in a straight line. This path is interrupted by the $\mathrm{AB}$ when it is placed between the source and the receiver. A portion of the transmitted acoustic energy is either reflected or scattered back towards the source, and other portion is transmitted through the barrier, diffracted from the barrier's edge or absorbed by the material of the barrier (see Fig. 1a). However, the use of $\mathrm{ABs}$ in urban areas is associated with several drawbacks: on one hand, the placement of continuous walls in cities presents aesthetic and communication problems related to both the breakdown of the cityscape and the physical isolation of the acoustically protected areas. On the other hand, the state of technology in the field of ABs nowadays does not guarantee a specific protection for each kind of noise because they are not able to distinguish the noise to be controlled, as because the same screen can be used to protect noises as different as a truck noise or an ambulance siren warning. These drawbacks make the use of the current ABs inappropriate in urban areas for noise control in buildings. 

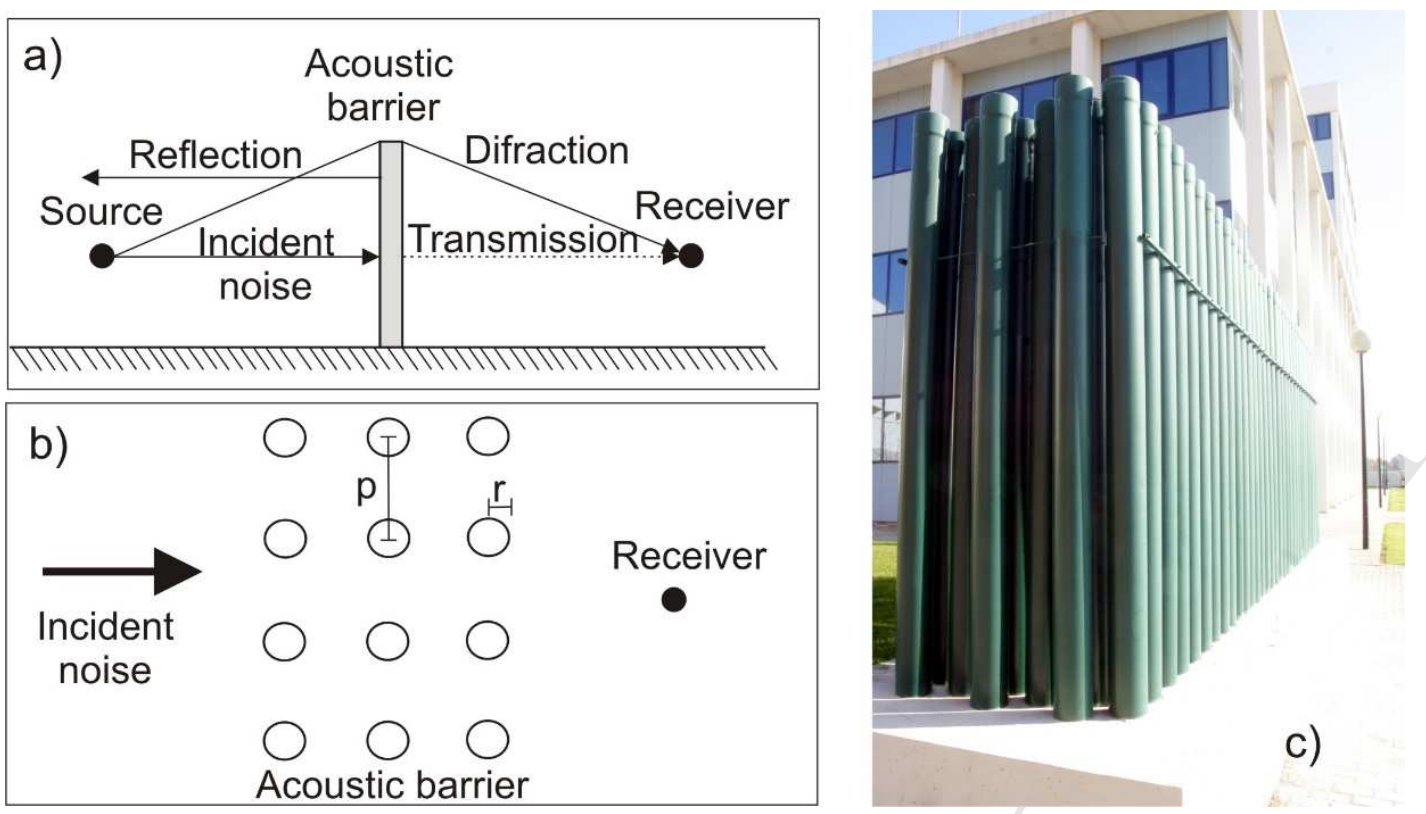

Figura 1.- (a) Acoustic performance of an AB; (b) Plan view of a Sonic Crystal with the main parameters of the array formed; (c) A picture of a SCAS made up with rigid cylinders as scatterers.

In the last decade, the discovery of new materials has enabled the development of new devices devoted to noise control. One of these materials is a composite generally called Sonic Crystal (SC), formed by periodic arrays of acoustic scatterers separated by a predetermined lattice constant and embedded in air. In Fig.1b we show a plan view of an SC with the main parameters of the array formed. The SC adds a new noise control mechanism based on the structuring of the scatterers which is different from what was previously known. Due to this new mechanism, there are ranges of frequencies where the wave propagation through SC is forbidden, called band gaps (BG), and their existence can be explained using a Bragg scattering process $[4,5]$. Both the size and the position of the BG in the frequencies domain basically depends on the geometrical characteristics of the array, the lattice constant $p$, and the radius of the cylinders $r$. As a consequence of the existence of $\mathrm{SC}$, a new generation of $\mathrm{ABs}$ has appeared with high technological procedures and with aesthetic conditions that allow their use as ABs in noise control of buildings in 
urban areas. The ABs based on SC are usually called Sonic Crystals Acoustic Screens (SCAS) and nowadays their use is at an intermediate point between the basic research of its physical properties and its widespread use as noise control devices. An example of SCAS can be seen in Fig.1(c). Among the properties of these devices in terms of noise control the following may be mentioned, (i) SCAS are discontinuous materials derived from a sculpture and, consequently, the aesthetic aspects are improved, at the same time giving visual continuity to the urban landscape [6]; (ii) from the technological point of view, SCAS allow the inclusion in their acoustic design of different noise control mechanisms as absorption or resonance together with the BG, intrinsic of these materials, such that all the mechanisms involved in the design of SCAS work constructively and separately. This fact is usually called tunability [7] and, as a consequence, the designer can choose the frequency range in which each of the noise control mechanisms must act, allowing the design of customized SCAS for each type of noise. These facts have led to the homologation of various SCAS that are acoustically competitive with respect to the current ABs [8]. One example in the use of SCAS can be seen in the Eindhoven A2 ring road, where Van Campen Industries have placed $22,000 \mathrm{~m}^{2}$ of SCAS with high acoustic performance, formed by rock wool cylinders wrapped with aluminium perforated sheets. Some images of this SCAS can be seen at [9]. In their design and construction, two noise control mechanisms are involved: BG and absorption.

Several theoretical approaches have been made to characterise the acoustic behaviour of SCAS, such as the Multiple Scattering Theory (MST) [10-12], the Plane Wave Expansion (PWE) [13], the Finite Difference Time Domain (FDTD) [14], the Finite Elements Method (FEM) [15] or the Method of Fundamental Solutions (MFS) [16]. However, the SCAS design is not easy due to the complex acoustic phenomenology involved, and the current theoretical models named above are not suitable because they have been developed 
for research purposes only. Thus, some restrictions such as the consideration of infinite arrays or the assumption of scatterers with infinite length are taken into account in these models, allowing the simplification of the problem by means of transforming a threedimensional (3D) case into a two-dimensional (2D) one, very distant from real situations. Admittedly, the numerical models developed enable the analysis of 3D cases, but usually at a high computational cost. Therefore, the development of design models reflecting the real working conditions of SCAS at a low computational cost seems necessary in order to meet the possible demand that installing this new kind of $\mathrm{AB}$ produces in the world of acoustic engineering. These models should consider all the acoustic phenomena related to the particular SCAS that is being designed, which means that the supposition of the real 3D case should be taken into account. In this sense, an overlapping model [17] developed using the Finite Elements Method (FEM) to design real SCAS has recently been presented. This model responds to the above requirements providing a designing tool to analyse real cases, which are easy to use and are at a low computational cost.

In this paper we present the implementation of the overlapping model mentioned above to the case of a new design of SCAS that are more powerful than the Eindhoven barrier named previously. In such SCAS we have considered three noise control mechanisms: BG, absorption and resonances besides considering diffraction at the upper edge of the screen. Several cases have been analyzed, adding different noise control mechanisms one by one to the scatterers, following the idea of tunability. We have named scatterers that are formed in this way 'multi-phenomena scatterers'. We also present a practical application of the model, where the diffraction at the upper edge of the device is reduced using the destructive interference mechanism in a predetermined range of frequencies. We provide numerical predictions obtained with the overlapping model, which is validated in the most 
complex case with experimental results. As an example, we have considered here a SCAS formed by an array of cylindrical multi-phenomena scatterers arranged in a square array. These systems are widely used in research due to several reasons such as their simplicity, their size or the high amount of symmetry of the cylinders $[8,17]$.

The paper is organized as follows: First, the experimental set-up used to validate the numerical results is presented. Afterwards, we explain the implementation of the overlapping model to the case analysed here. Numerical results as well as the comparison with the experimental ones are analysed and discussed in the next section. Finally, the last section contains the concluding remarks, where the results are summarised.

\section{EXPERIMENTAL CHARACTERIZATION}

To validate the numerical results obtained by means of our model, a set of experiments have been performed in an echo-free chamber of size $8 \times 6 \times 3 \mathrm{~m}^{3}$, which simulate free field conditions. A prepolarised free-field 1/2" microphone Type 4189 B\&K located at $0.11 \mathrm{~m}$ from the sample and a directional sound source GENELEC 8040A emitting continuous white noise, located $1 \mathrm{~m}$ behind the sample in order to consider the wave impinging on the sample as a plane wave, have been used throughout the experiments. A SCAS made of 16 (4 columns $\mathrm{x} 4$ rows) multi-phenomena scatterers arranged in a square array with lattice constant $\mathrm{p}=0.33 \mathrm{~m}$ is considered, as one can see in fig.2(a). With these parameters the first BG at $0^{0}$ incidence of the wave on the SCAS appears at a frequency $\mathrm{f}=515 \mathrm{~Hz}$.

The design of the cylindrical scatterers has been made following the guidelines proposed by Romero-Garcia et al. [7], successfully used to standardise an advanced model of SCAS 


\section{ACCEPTED MANUSCRIPT}

[8] following the European standards [18-20]. The scatterers are formed by PVC cylindrical resonant cavities with inner radius $\mathrm{r}_{0}=0.095 \mathrm{~m}$, thickness of the rigid wall $\mathrm{e}_{1}=0.005 \mathrm{~m}$, and a slot along their entire length with aperture $\mathrm{d}=0.02 \mathrm{~m}$. These rigid cylinders are wrapped by a layer of rock wool as absorbent material, with thickness $e=0.04$ $\mathrm{m}$. As a result, cylindrical multi-phenomena scatterers with external radius $\mathrm{r}=0.14 \mathrm{~m}$ and with length $\mathrm{h}=1.20 \mathrm{~m}$ are considered in the construction of the SCAS checked here. Geometrical details of these scatterers are shown in the inset of fig.2(a).

On the other hand, and with the idea of reducing the diffraction at the upper edge of the cylinders that form the SCAS, a set of 4 empty PVC cylinders with radius $r_{1}=0.05 \mathrm{~m}$ and wall thickness $\mathrm{e}_{1}=0.005 \mathrm{~m}$ will be arranged with a separation distance between them equal to the lattice constant of the SCAS, $\mathrm{p}=0.33 \mathrm{~m}$ and located at $\mathrm{h}_{1}=0.33 \mathrm{~m}$ height on the top of the sample, as can be seen in fig. 2(a). A photograph of the entire device along with the position of the microphone can be seen in fig.2(b).
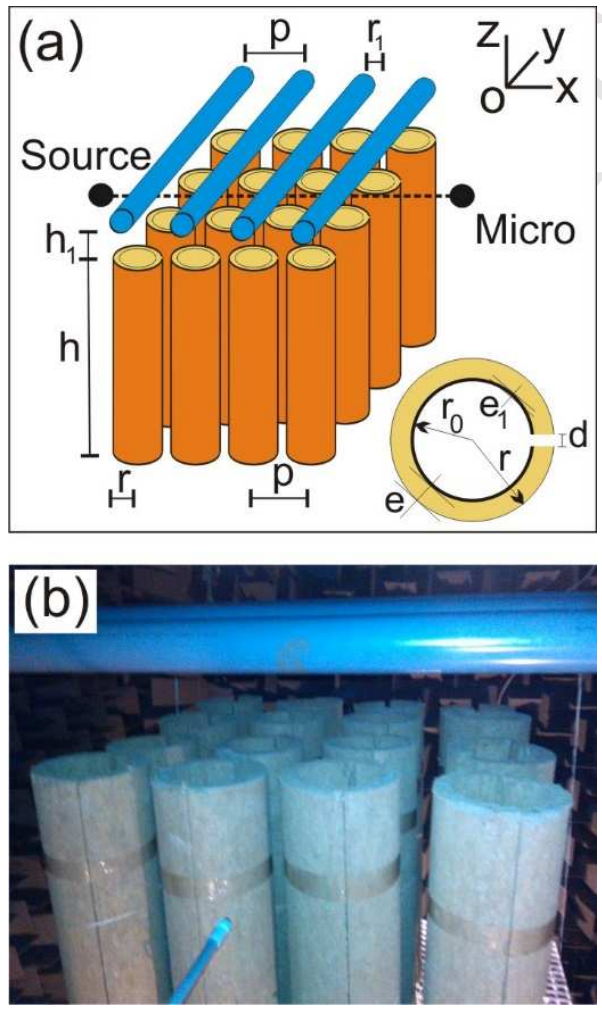

Figure 2.- (a) Scheme of the experimental set-up used; (b) A picture of the sample made of multi-phenomena cylindrical scatterers. A partial view of the cylinders used to reduce diffraction at the upper edge of the sample is also shown. 


\section{NUMERICAL OVERLAPPING DESIGN MODEL}

As we have explained previously, the design model presented here is based on the idea of tunability, which means that the different noise control mechanisms involved in the problem can be separately treated. This fact allows us to split the real three dimensional problem into some two dimensional analyses, which are easier to study without interferences from the others and at a low computational cost. Finally, the resulting total complex acoustic pressure of the three dimensional case can be calculated by combining the partial complex pressures obtained at each one of the two dimensional cases. A complete explanation of the model is presented in reference [17]. To develop the model and to obtain the numerical predictions, the commercial software COMSOL Multiphysics has been used throughout the study.

To illustrate this methodology, we have modelled the proposed SCAS explained in the previous section. We will present different scatterer configurations, progressively adding new noise control mechanisms, specifically resonances and absorption. This variation is easy to implement in our model simply by activating or deactivating different domains contained therein, as we will see in the next section. In our analysis, the complete geometries of both two dimensional cases, including all the mechanisms considered, are shown in fig. 3(a) and fig. 3(b), with the different mechanisms involved assigned to each one of them. On one hand, the mechanisms related to the composition of the scatterers (BG, absorption and resonances) have been assigned to the geometry shown in fig. 3(a). This assignment can be easily understood taking into account that the composition of the scatterers remains unchanged in the direction of the axes of the cylinders $(\mathrm{OZ}$ direction in our model), and the system could be considered as a two dimensional case (OXY plane). 
We have called this first geometry "plan view". As one can see, the geometry is formed by a section of the SCAS considered, perpendicular to the axes of the cylindrical scatterers, so that they are represented by circles including all the noise control mechanisms considered. In the inset of fig. 3(b) one can observe the composition of the multi-phenomena cylinders, which are formed by empty rigid cylinders made of PVC with wall thickness $\mathrm{e}_{1}$ and radius $\mathrm{r}_{0}$. Next, a layer of an absorbent material (rock wool) with external radius $\mathrm{r}$ and thickness e is wrapped around the rigid cylinder. To simulate the slot along their length in the numerical model, a rectangle of height $d$ is inserted across the scatterer. The different parts of this geometry can be activated or deactivated in the model to simulate different materials or different compositions of the cylindrical scatterers. On the other hand, diffraction at the upper edge of the cylindrical scatterers has been included in the geometry shown in fig. 3(b). We have called this geometry "sectional view". In this case the two dimensional model is formed by a sectional plane parallel to the axes of the cylindrical scatterers (OXZ plane), and they are represented by rectangles with their bases equal to the diameter of the cylindrical scatterers [17]. One can observe the layers of the absorbent material in yellow colour.

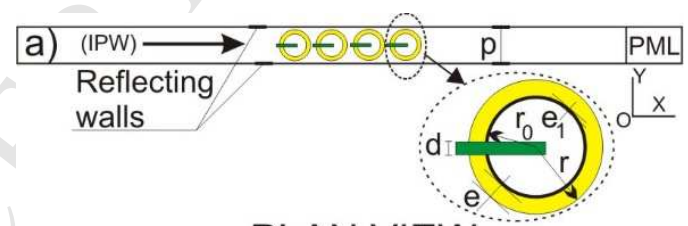

PLAN VIEW

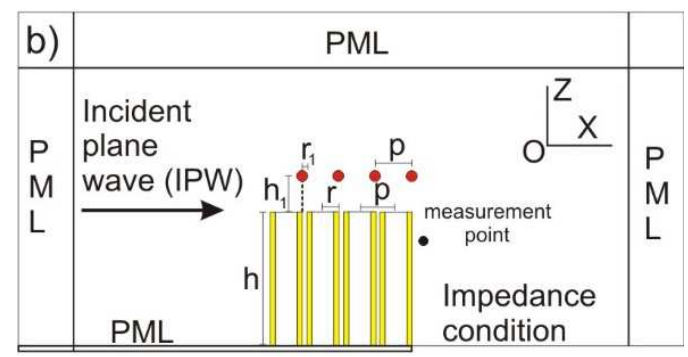

SECTIONAL VIEW

Figure 3.- Overlapping design model where the real three dimensional problem is replaced by two two dimensional cases; (a) Plan view (OXY, two dimensional case), which 
includes BG, resonances and absorption mechanisms. One can see the OXY section of the multi-phenomena cylindrical scatterers, as well as the reflected lateral lines and the PMLs that define the boundaries of the domain. In the inset, one can see the main geometrical parameters of the multi-physical cylindrical scatterers; (b) Sectional view (OXZ, two dimensional case) with the main geometrical parameters of the problem considered. In this two dimensional model, only diffraction at the upper edge of the scatterers is considered, and one can see the OXZ section of the real three dimensional problem. The PMLs and the impedance condition defined at the boundaries are also indicated in the figure.

To develop the second objective of the paper, which is the practical use of our model to design a solution to reduce the diffraction at the upper edge of the SCAS using destructive interferences, a set of empty rigid cylinders (red colour in fig. 2(b)) with radius $r_{l}$ and wall thickness $e_{1}$ is included in the model, located perpendicularly and on the top of the cylindrical scatterers. We have called them tuned solution. These cylinders are viewed in the sectional view as a set of circles, and they are arranged near the back boundary of each multi-phenomena scatterer in order to reduce the diffraction. The distance $\mathrm{h}_{1}$ between these cylinders and the cylindrical scatterers has been chosen to decrease diffraction at a frequency $\mathrm{f}=515 \mathrm{~Hz}$, which is the Bragg's frequency of the first band gap.

The geometrical domain in fig.3(a) where the plan view of the SCAS considered is represented and confined by two completely reflected lines at the upper and lower boundaries and separated by the lattice constant of the array $p$. This artifice produces the effect of a semi-infinite array of cylinders, and has been successfully used in several analysis related to $\mathrm{SC}[7,21]$, allowing the decrease of the computational cost by reducing the numerical domain. On the other hand, to consider only diffraction an impedance 
continuity is considered at the lower boundary behind the array of cylindrical scatterers in the sectional view represented in fig.3(b) in order to simulate free transmission conditions to avoid reflections on the ground behind the SCAS. Finally, the rest of the boundaries of both sections are surrounded by Perfectly Matched Layers (PML) to simulate the Sommerfeld radiation conditions in the numerical resolution of scattering problems [22].

Regarding the modelling of the different elements that form the proposed SCAS, we have considered the PVC cylinders as acoustically rigid, and therefore the Neumann boundary conditions (zero sound velocity) are applied to their surfaces. On the other hand, we have modelled the rock wool as a porous material using the Delany-Bazley model implemented in COMSOL, where the flow resistance takes the value of $\mathrm{R}=23,000 \mathrm{~Pa} \cdot \mathrm{s} \cdot \mathrm{m}^{-2}$.

With these conditions, we have numerically calculated the total complex acoustic pressure for each two dimensional model, $p_{t}$, which can be expressed as

$$
p_{t}=p_{o}+p_{s}
$$

where $p_{o}$ is the complex acoustic pressure of the incident wave given by the starting conditions of the problem, and $p_{s}$ is the scattered complex pressure given by the scattered wave produced inside the domain due to the interaction between the incident wave and both the geometry and the physical properties of the sample. The characteristics of the mesh and the number of degrees of freedom will depend on the case considered, and will be explained in each of the problems solved. Taking into account that both the SCAS used in the numerical calculations and in the experiments have the the same geometrical characteristics, it is possible to compare them to validate the numerical model developed. 
However, some discrepancies can appear in the comparison due to the finite size of the experimental sample.

To acoustically contrast the different devices proposed, we have calculated the acoustic attenuation at a point located with coordinates $(0.11,0,-0.30) \mathrm{m}$ from the back edge of the last cylinder that form the 2DSC (see fig.3(b)). The reason for deciding that this point should be so close to the SCAS is for checking the effect of the diffracted wave. The acoustic attenuation is given by the Insertion Loss (IL) parameter, defined as the difference between the sound level at a point recorded with and without the sample. Considering the overlapping model proposed and following reference [17], the IL for the general case can be written as

$$
\mathrm{IL}=20 \log _{10}\left|\frac{\mathrm{p}_{\text {direct }}}{\mathrm{p}_{\text {interfered }}}\right|=20 \log _{10}\left|\frac{\mathrm{p}_{\mathrm{o}}}{2 \mathrm{p}_{\mathrm{o}}+\mathrm{p}_{\mathrm{s}_{(\mathrm{BRAGG}+\mathrm{RES}+\mathrm{ABS})}}+\mathrm{p}_{\mathrm{s}_{(\mathrm{DIF})}}}\right|
$$

where $p_{o}$ is the complex incident pressure used at each one of the two dimensional models, $p_{S(B R A G G+A B S+R E S)}$ is the complex scattered pressure obtained in the plan view model, due to the BG (BRAGG), resonances (RES) and absorption (ABS) noise control mechanisms in the most complex case, and $p_{s(D I F)}$ is the complex scattered pressure due to diffraction and provided by the sectional view model. On the other hand, $\left(p_{o}+p_{S(B R A G G+R E S+A B S)}\right)$ and $\left(p_{o}+p_{s(D I F)}\right)$ are the complex interfered pressures in both two dimensional models. Note that the adaptation of equation (2) to our case has been made taking into account the specific characteristics of the software used. Thus, the global complex incident pressure that impinges on the SCAS is $p_{o}$ ( $p_{\text {direct }}$ in equation (2)), the complex interfered pressures, $p_{\text {interfered, }}$, which are defined for both the Sectional and the Plan views, are obtained by 
adding the incident complex pressure po to the complex scattered pressure obtained in each case $\left(p_{s(B R A G G+R E S+A B S)}\right.$ for the Plan view $2 \mathrm{D}$ model, and $p_{s(D I F)}$ for the Sectional view 2D model).

Note that to obtain both IL (with and without the tuned solution for diffraction proposed) it is enough to recalculate only the complex pressure of the sectional view where the tuned solution is implemented because the plan view does not change. This fact enables us to reduce even more both the complexity of the problem and the computational cost, because it is possible to work only with the two dimensional case where the acoustic phenomenon is being treated. Finally, we present the IL result in narrow band to check the position and the attenuation provided by each of the noise control mechanisms considered.

\section{RESULTS AND DISCUSSION}

Using the overlapping model, in this section we have analysed the IL of the different SCAS formed, including the different noise control mechanisms selected in each case. We present three cases, in which we assume the finite length of the cylindrical scatterers in order to consider real three dimensional conditions: (i) SCAS formed by rigid (PVC) cylinders as scatterers. In this case, there are two mechanisms involved: BG and diffraction at the upper edge of the cylinders; (ii) SCAS formed by rigid (PVC) cylinders with a longitudinal slot. Here there are three mechanisms implicated: BG, resonances and diffraction; (iii) SCAS formed by a rigid (PVC) cylinder slotted and wrapped in a layer of porous material (rock wool). There are four acoustic mechanisms acting in this case: BG, resonances, absorption and diffraction. In all cases we have also implemented the tuned solution to reduce the diffraction at the upper edge of the SCAS using the destructive interference mechanism. In the latter case, where a high number of acoustic mechanisms 
are involved, we have checked the numerical simulations with experimental results stated in order to verify the proper operation of the model.

The adaptation of the proposed model to the SCAS formed by rigid cylinders can be seen in fig.4(a). One can observe the different domains activated (red colour): on one hand, the rigid wall and the porous material ( $e_{1}$ and $e$ thickness respectively) are working as rigid materials to simulate the BG mechanism, taking into account that the thickness of the wall in rigid materials is not important in the Bragg scattering phenomenon. These activated layers can be seen in both two dimensional analyses (plan and sectional views). Note that the position of the BG peak in the frequencies domain can be changed by simply varying the geometrical characteristics of the array of scatterers. On the other hand, to check the validity of the solution proposed to reduce diffraction, we have activated the red circles that constitute the tuned solution. The plan view case has been solved considering $1.02 \cdot 10^{5}$ degrees of freedom and $8.40 \cdot 10^{4}$ elements and in the sectional case, $1.63 \cdot 10^{5}$ degrees of freedom and $1.20 \cdot 10^{5}$ elements have been necessary to solve it. Here, the computational time to obtain IL solutions is $4500 \mathrm{~s}$.

With these settings applied to the generalised overlapping comprehensive model, we have numerically calculated the IL spectra with and without the tuned solution adopted to reduce diffraction by means of the generalised expression (2) adapted to this case:

$\mathrm{IL}=20 \log _{10}\left|\frac{\mathrm{p}_{\text {direct }}}{\mathrm{p}_{\text {interfered }}}\right|=20 \log _{10}\left|\frac{\mathrm{p}_{\mathrm{o}}}{2 \mathrm{p}_{\mathrm{o}}+\mathrm{p}_{\mathrm{s}_{(\mathrm{BRAGG})}}+\mathrm{p}_{\mathrm{s}_{(\mathrm{DIF})}}}\right|$ 
The IL results can be seen in fig.4(b). One can observe, in both spectra, the existence of the first $\mathrm{BG}$ centred at $\mathrm{f}=515 \mathrm{~Hz}$. The results with (red dotted line) and without (black continuous line) the tuned solution are compared, and one can observe the increase of the IL for the case of the tuned solution around the Bragg's frequency ( $\mathrm{f}=515 \mathrm{~Hz}$ ), which means that the design proposed to reduce diffraction works suitably.

Next we have applied the proposed model to the case of a SCAS formed by rigid and slotted cylindrical scatterers. In this case, we have added the existence of resonant cavities at each one of the multi-phenomena scatterers. The adaptation of the overlapping model to this case has been easy: the rectangle of height $d$ that creates the slot of the rigid cylinders shown in the plan view (fig3) has been activated, creating a resonator. This approach can be seen in fig.4(c). Again, we have calculated the IL spectra with and without the tuned solution. In this case, the general expression (2) can be written as:

$$
\mathrm{IL}=20 \log _{10}\left|\frac{\mathrm{p}_{\text {direct }}}{\mathrm{p}_{\text {interfered }}}\right|=20 \log _{10}\left|\frac{\mathrm{p}_{\mathrm{o}}}{2 \mathrm{p}_{\mathrm{o}}+\mathrm{p}_{\mathrm{s}_{(\mathrm{BRAGG}+\mathrm{RES})}}+\mathrm{p}_{\mathrm{s}_{(\mathrm{DIF})}}}\right|
$$

The IL spectra are represented in fig.4(d) where the high quality of the results is shown. In both cases it is possible to see the resonance peak that appears around $\mathrm{f}=200 \mathrm{~Hz}$ due to the geometrical characteristics of the resonator proposed, and the existence of the BG around $\mathrm{f}=515 \mathrm{~Hz}$, showing the good performance of both mechanisms according to the tunability idea. Note that the position of the resonance peak can be moved in the frequencies range by changing the geometrical characteristics of the resonator created. Moreover, in fig.4(d) one can see the increase of the IL when the tuned solution is activated (red dotted line) compared with the case where the tuned solution is deactivated (black continuous line). 

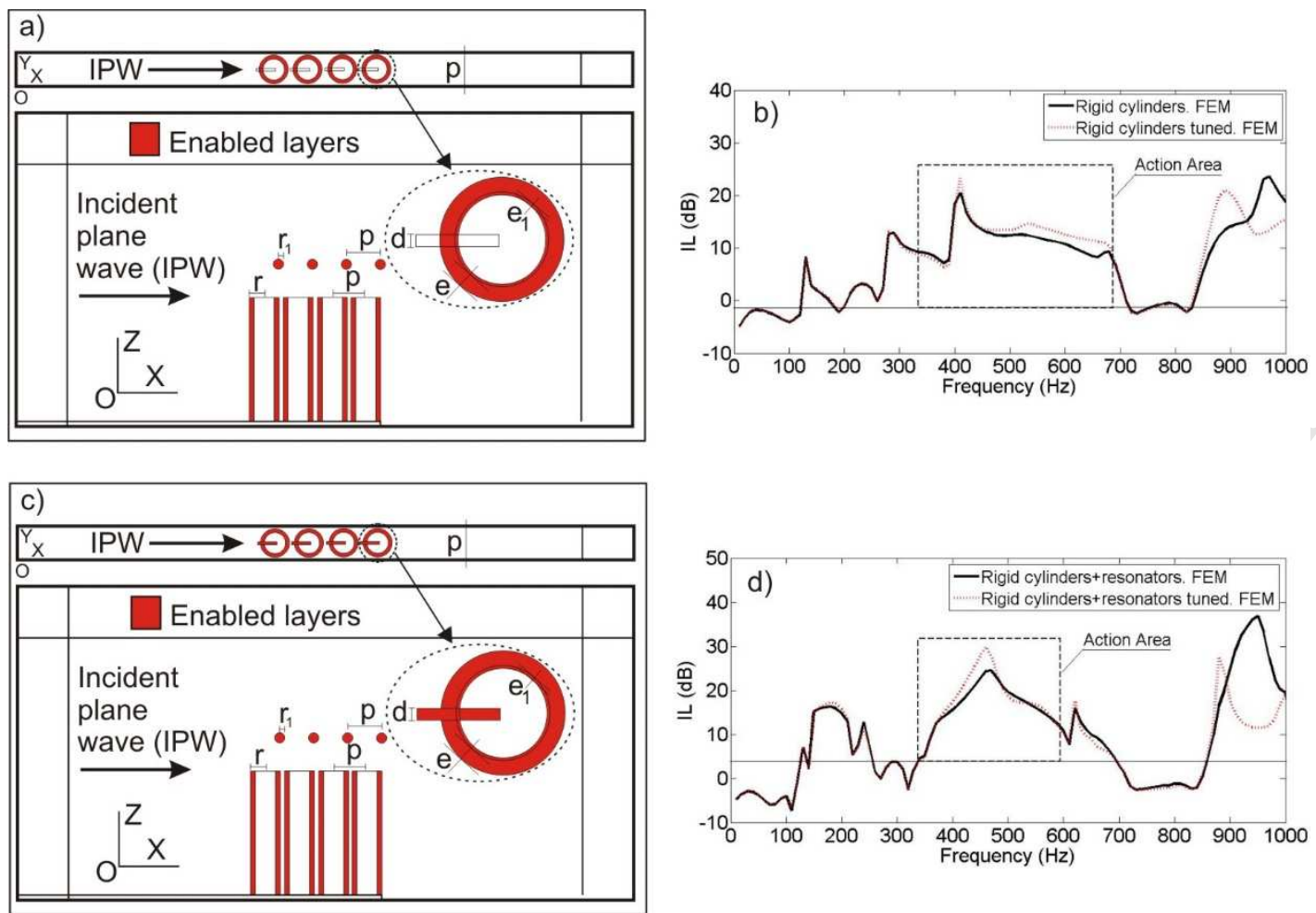

Figure 4.- Overlapping model applied to two different SCAS cases (plan and sectional views). (a) and (c) show the overlapping model for the design of SCAS formed, respectively, by rigid cylinders or rigid slotted cylinders as scatterers; (b) and (d) represent the corresponding IL spectra for both cases in the range of frequencies analysed with (red dotted line) and without (black continuous line) the tuned solution proposed to reduce the diffraction at the upper edge. The squares formed by the black dotted line in both spectra define the limits of performance of the proposed tuned solution.

To numerically solve this problem, $1.5 \cdot 10^{5}$ elements with $6,51 \cdot 10^{5}$ degrees of freedom for the plan view case, and $5.1 \cdot 10^{5}$ elements with $3.02 \cdot 10^{5}$ degrees of freedom in the sectional view case have been used. In this case, the computational cost is $6000 \mathrm{~s}$.

Finally, we have analysed the case of a SCAS formed by rigid cylindrical slotted scatterers with absorbent material. To do that, we have added a layer of absorbent materials (rock wool) in the cylindrical scatterers so that four acoustic mechanisms are involved. In this 
instance, the adaptation of the overlapping model reflects the changing of the properties of the external ring of the cylindrical scatterers with thickness e: from the supposition of rigid material adopted in the previous cases to the assumption of porous material with the properties explained above, as is shown in fig.5(a). An example of the real pressure solution in the complete domain of both plan and sectional views for the Bragg's frequency $(\mathrm{f}=515 \mathrm{~Hz})$ are shown in fig.5(b).
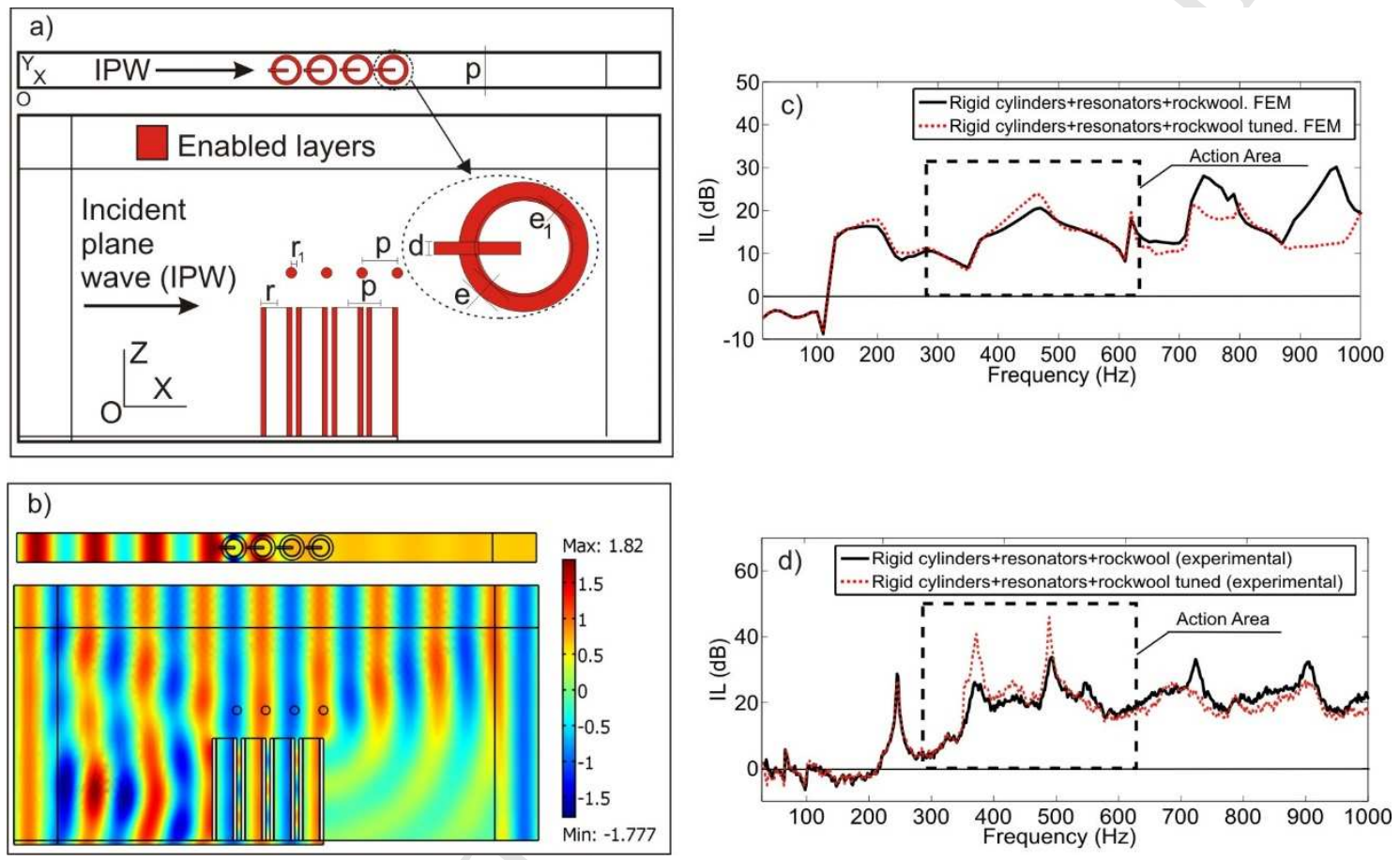

Figure 5.- Overlapping model applied to the case where 4 noise control mechanisms are considered (BG, resonances, absorption and diffraction at the upper edge of the SCAS). (a) Scheme of the two dimensional cases (plan and sectional views) in which the real three dimensional problem has been split. The domains activated are represented in the red colour; (b) Simulated real values of the total pressure of the complete domain at $(f=515$ $\mathrm{Hz}), \operatorname{Re}\left(p_{o}+p_{s}\right)$, are mapped for both the plan and sectional views; (c) and (d) Numerical and experimental IL spectra respectively at the tested point with (red dotted line) and without (black continuous line) the tuned solution proposed to reduce diffraction at the upper edge of the considered SCAS. 
Again, we have obtained numerical outcomes of the IL spectra using the overlapping model with and without the tuned solution for diffraction by means of the expression (2). The results can be seen in fig.5(c). Once more one can observe the increase of the IL in the range of frequencies chosen when the tuned solution is activated. However, in this case one can see a decreasing of the IL around $1000 \mathrm{~Hz}$. This reduction can be explained in terms of the constructive interferences produced at this frequency due to the geometry of the tuned solution adopted, which is designed to reduce diffraction only at $\mathrm{f}=515 \mathrm{~Hz}$. Note that the acoustic effect of the rock wool could be changed by varying the geometrical or absorbing properties of the material. These results have been experimentally verified and the results can be seen in fig.5(d), where one can check the numerically obtained results. Note that in the experimental set up we have used a directional sound source, but a plane wave has been considered in the overlapping model developed. This apparent contradiction can be explained in terms of the size of the anechoic chamber, where the assumption of plane wave cannot be considered in the obtaining of the experimental results. Moreover, this experimental set up have been used successfully throughout last years, providing experimental results in good agreement with the numerical or analytical predictions obtained using either plane wave or punctual source [5]. These last results enable us to experimentally validate the generalized model proposed. Because of the difficulties to obtain the experimental results due to the measuring point is very near of the sample where the output wavefront is not completely formed, and also due to the finite size of the sample, this validation is not based the accurate reproduction of the shape of the experimental IL spectrum using our overlapping model, but in the check that (i) both numerical and experimental spectra have the attenuation bands due to the different acoustic phenomena involved ( $\mathrm{BG}$, absorption and resonances) in the same position in the range of 
frequencies, (ii) the IL level for numerical and experimental cases in $\mathrm{dB}$, and (iii) the IL increases in both cases when the tuned solution is enabled. In this case, $4.37 \cdot 10^{5}$ elements with $7.89 \cdot 10^{5}$ degrees of freedom for the plan view case, and $5.1 \cdot 10^{5}$ elements with $3.02 \cdot 10^{5}$ degrees of freedom for the sectional view case have been used to solve the complete problem. The computational time employed here is $6100 \mathrm{~s}$.

Finally, to get an idea about the reduction of the computational time in our model, we have obtained some IL spectra with different FEM models controlling the time cost. In the case of a real 3D model formed by a SCAS formed by $16(4 \times 4)$ multi-phenomena scatterers, the computational cost has been around 20 days. If we use the 3D reduced numerical model (developed with FEM) proposed by us in reference [10], the computational cost is around 5 hours. Finally, for our overlapping model this cost is around 1.5 hours. Note that this last computational time can be reduced because it is not necessary to recalculate both $2 \mathrm{D}$ models in all cases: if, for example, we want to analyse some solutions to reduce diffraction at the upper edge, we only have to recalculate the $2 \mathrm{D}$ sectional view, because the $2 \mathrm{D}$ plan view not change. Thus, it is possible to reduce much more the computational time in our overlapping model.

\section{CONCLUSIONS}

An application of an overlapping model to design SCAS formed by cylindrical scatterers embedded in air, taking into account the complex acoustic phenomenology that appears in the real use of this new kind of barrier, is presented in this paper. SCAS can be used in noise control in buildings to reduce the most important type of noise that appears in cities, i.e. the transport noise, for aesthetic and technological reasons due to the fact that these barriers come from a sculpture and are versatile enough to be 
designed for specific noises. SCAS are technologically advanced and acoustically competitive with respect to classical $\mathrm{AB}$, and therefore the versatile overlapping model presented is necessary to design this new generation of $A B$ that have already appeared on the noise control market. The model is based on the idea of tunability and allows the analysis of each one of the noise control mechanisms involved independently and, at the same time, can be used to find the best solution for each case without increasing the computational cost, selecting the range of frequencies in which the designer wants each noise control mechanism to work. The numerical simulations presented, supported by accurate experimental results allow us to validate the model. Moreover, the model enables the inclusion of any new noise control mechanism desired, with the only requiring caution when introducing it into the appropriate two dimensional model in which the complete three dimensional model has been split.

Acknowledgement

This work is supported by MEC (Spanish Government) under grant No. MTM201236740-C02-02.

\section{REFERENCES}

[1] EC Directive, (2002), Directive 2002/49/EC of the European Parliament and of the Council of 25 June 2002 relating to the assessment and management of environmental Noise, Official Journal of the European Communities (18.7.2002), Brussels

[2] Schafer, R. (1977). The Tuning of the World, Borzoi book (Knopf). 
[3] Department of Transportation, Federal Highway Administration U.S.A. (2001), Keeping the Noise Down. Highway Traffic Noise Barriers, Washington, 2001 FHWA-EP-01-004 HEPN/2-01 (10M) E.

[4] Sigalas M.M. and Economou E.N., J. Sound Vib., 158 (1992) 377.

[5] Sánchez-Pérez J.V., Caballero D., Martínez-Sala R., Rubio C., Sánchez-Dehesa J., Meseguer F., Llinares J. and Gálvez F., Phys. Rev. Lett., 80 (1998) 5325. http://dx.doi.org/10.1103/PhysRevLett.80.5325

[6] Martínez-Sala R., Sancho J., Sánchez-Pérez J.V., Gómez V., Llinares J. and Meseguer F., Nature, 378 (1995) 241. doi:10.1038/378241a0

[7] Romero-García V., Sánchez-Pérez J.V. and Garcia-Raffi L.M., J. Appl. Phys., 110 (2011) 014904. http://dx.doi.org/10.1063/1.3599886

[8] Castiñeira-Ibáñez S., Rubio C., Romero-García V., Sánchez-Pérez J.V. and GarciaRaffi L.M., Arch. Acoust, 37 (2012) 455. doi: 10.2478/v10168-012-0057-9,

[9] Van Campen Industries. [On Line] Available in http://www.campen.nl/albumsound-absorbent-noise-barriers [7 September, 2009].

[10] Linton, C. M. and Evans, D. V. J. Fluid Mech, 215 (1990) 549.

http://dx.doi.org/10.1017/S0022112090002750

[11] Martin, P.A. Multiple Scattering: Interaction of Time-Harmonic Waves with N Obstacles Cambridge University Press, Cambridge, (2006).

[12] Chen, Y. Y. and Ye, Z, Phys. Rev. E, 64, 036616, (2001). http://dx.doi.org/10.1103/PhysRevE.64.036616

[13] Kushwaha, M. S., Appl. Phys. Lett. 70, 3218, (1997). http://dx.doi.org/10.1063/1.119130

[14] Taflove A., Soukoulis C.M. Advances in Computational Electrodynamics: The Finite Difference Time Domain Method. Artech House, London (1998). 
[15] Ihlenburg F., Marsdem J. E. and Sirovich L. Finite Element Analysis of Acoustic Scattering, Springer Verlag, New York Inc, (1998).

[16] P. G. Santos, J. Carbajo, L. Godinho, J. Ramis. . Boundary Elements and Other Mesh Reduction Methods XXXVII, C.A. Brebbia, Wessex Institute of Technology, UK and A.H-D. Cheng, University of Mississippi, USA. DOI: 10.2495/BE370191, (2014)

[17]Castiñeira-Ibáñez S., Rubio C., and Sánchez-Pérez J.V., EPL, 101 (2013) 64002. doi:10.1209/0295-5075/101/64002

[18] European Committee for Standardisation, EN 1793- 1 (1997), Road Traffic Noise Reducing Devices - Test Method for Determining the Acoustic Performance - Part 1: Intrinsic characteristics of sound absorption, CEN, Brussels, Belgium.

[19]European Committee for Standardisation, EN 1793- 2 (1997), Road Traffic Noise Reducing Devices - Test Method for Determining the Acoustic Performance - Part 2: Intrinsic characteristics of airborne sound insulation under diffuse sound field conditions, CEN, Brussels, Belgium.

[20]European Committee for Standardisation, EN 1793- 3 (1997), Road Traffic Noise Reducing Devices - Test method for Determining the Acoustic Performance - Part 3: Normalised Traffic Noise Spectrum, CEN, Brussels, Belgium.

[21] Gupta A., Lim K.M. and Chew C. H., J. Acoust. Soc. Am., 132 (2012) 2909. doi: $10.1121 / 1.4744930$

[22] Berenguer, J.P., J. Compt. Physics, 114 (1994) 185. 


\section{FIGURES CAPTIONS}

Figura 1.- (a) Acoustic performance of an AB; (b) Plan view of a Sonic Crystal with the main parameters of the array formed; (c) A picture of a SCAS made up with rigid cylinders as scatterers.

Figure 2.- (a) Scheme of the experimental set-up used; (b) A picture of the sample made of multi-phenomena cylindrical scatterers. A partial view of the cylinders used to reduce diffraction at the upper edge of the sample is also shown.

Figure 3.- Overlapping design model where the real three dimensional problem is replaced by two two dimensional cases; (a) Plan view (OXY, two dimensional case), which includes BG, resonances and absorption mechanisms. One can see the OXY section of the multi-phenomena cylindrical scatterers, as well as the reflected lateral lines and the PMLs that define the boundaries of the domain. In the inset, one can see the main geometrical parameters of the multi-physical cylindrical scatterers; (b) Sectional view (OXZ, two dimensional case) with the main geometrical parameters of the problem considered. In this two dimensional model, only diffraction at the upper edge of the scatterers is considered, and one can see the OXZ section of the real three dimensional problem. The PMLs and the impedance condition defined at the boundaries are also indicated in the figure.

Figure 4.- Overlapping model applied to two different SCAS cases (plan and sectional views). (a) and (c) show the overlapping model for the design of SCAS formed, respectively, by rigid cylinders or rigid slotted cylinders as scatterers; (b) and (d) represent the corresponding IL spectra for both cases in the range of frequencies analysed with (red 
dotted line) and without (black continuous line) the tuned solution proposed to reduce the diffraction at the upper edge. The squares formed by the black dotted line in both spectra define the limits of performance of the proposed tuned solution.

Figure 5.- Overlapping model applied to the case where 4 noise control mechanisms are considered (BG, resonances, absorption and diffraction at the upper edge of the SCAS). (a) Scheme of the two dimensional cases (plan and sectional views) in which the real three dimensional problem has been split. The domains activated are represented in the red colour; (b) Simulated real values of the total pressure of the complete domain at $(\mathrm{f}=515$ $\mathrm{Hz}$ ), $\operatorname{Re}\left(p_{o}+p_{s}\right)$, are mapped for both the plan and sectional views; (c) and (d) Numerical and experimental IL spectra respectively at the tested point with (red dotted line) and without (black continuous line) the tuned solution proposed to reduce diffraction at the upper edge of the considered SCAS. 


\section{Highlights:}

Technologically advanced Sonic Crystal Acoustic Screens are proposed.

An overlapping model to design these real three-dimensional screens is present.

The model presented enables the inclusion of any new mechanism of noise control. 